\title{
STRESS SENSITIVITY ANALYSIS OF THE BEAM AND SHELL FINITE ELEMENTS
}

The stress sensitivity analysis in conjunction with finite elements method represents an important tool for the influence analysis of the structural parameters. This analysis plays a significant role in the decision process of the formulation of the structural optimizing or probability analysis. The goal of the paper is to present theoretic and numerical aspects of the beam and shell element stress sensitivity analysis with the respect to the cross section parameters (cross section area, thickness, etc.). The whole computational procedure was inbuilt into Matlab's software module MATFEM.

Keywords: stress sensitivity analysis, beam, thin shell element, Matlab

\section{Introduction}

Nowadays the sensitivity analysis is a significant tool helping to realize a structural parameters influence analysis. This analysis is usually very computer time consuming but the results are very innovative. This process is often applied to a structural analysis, i.e. in stress and strain analysis, modal and spectral or buckling analysis, stochastic analysis and so on [3,6].

Application of the sensitivity analysis is not associated only with the structural optimizing but also with the analysis of the mechanical systems with uncertain parameters, mainly in the usage of so-called perturbation methods based on differentiation of the response with respect to the uncertain system parameters (stiffness, mass, damping, etc.). Implementation of this computational process into the finite element method characterized mainly the era of development of structural optimizing techniques in the eighties.

\section{Stress sensitivity analysis for beam finite element}

We will consider classic linear two-nodes beam element with a constant cross section (There is more information in $[1,5]$.).

Let's consider only a well-known linear distribution of the normal stress, i.e.

$$
\sigma_{x}=\frac{N_{x}}{A}-\frac{M_{o z}}{J_{z}} \cdot y+\frac{M_{o y}}{J_{y}} \cdot z,
$$

where $N_{x}$ is the internal axial force, $M_{o z}$ and $M_{o y}$ are bending moments, $A$ is the element cross-section area, $J_{z}$ and $J_{y}$ are moments of inertia $[2,5,7,8]$.

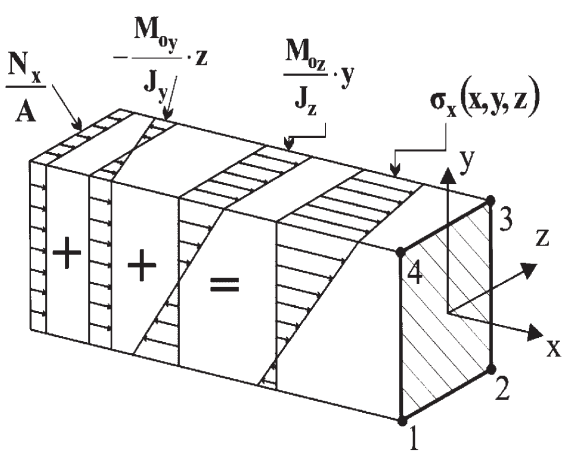

Fig. 1 Stress distribution in beam

If the bending moment is $[1,6]$

$$
M_{o}(x)=\left[\left(1-\frac{x}{l}\right) \frac{x}{l}\right] \cdot\left[\begin{array}{l}
M_{o 1} \\
M_{o 2}
\end{array}\right],
$$

than the normal stress function is following

$$
\begin{aligned}
& \sigma_{x}(x, y, z)=\left\{\begin{array}{llll}
0 & 0 & 0 & 0
\end{array}\left[\frac{z}{J_{y}} \cdot\left(1-\frac{x}{l}\right)\right]\left[-\frac{y}{J_{z}} \cdot\left(1-\frac{x}{l}\right)\right] .\right. \\
& \left.\cdot\left(\frac{1}{A}\right) 0000\left(\frac{z}{J_{y}} \cdot \frac{x}{l}\right)\left(-\frac{y}{J_{z}} \cdot \frac{x}{l}\right)\right\} \text {. } \\
& \cdot\left[N_{x 1} T_{y 1} T_{z 1} M_{k 1} M_{y 1} M_{z 1} N_{x 2} T_{y 2} T_{z 2} M_{k 2} M_{y 2} M_{z 2}\right]^{T}
\end{aligned}
$$

In agreement with points in Fig. 1 it is possible to write the relationship between normal stress in these marginal points of the cross-section and internal elements forces and moments, i.e.

\footnotetext{
* Milan Saga, Milan Vasko

Department of Applied Mechanics, Faculty of Mechanical Engineering, University of Zilina, E-mail: milan.saga@fstroj.uniza.sk
} 


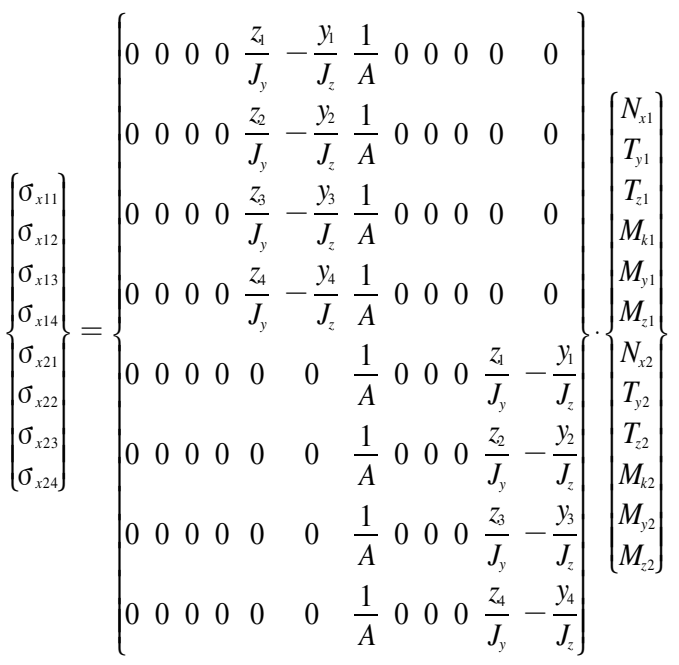

or

$$
\boldsymbol{\sigma}_{L}^{j}=\mathbf{C}_{L}^{j} \cdot \mathbf{f}_{L}^{j},
$$

The stress sensitivity centre is the derivative (5) with respect to a design variable $X_{i}$, i.e.

$$
\frac{\partial \boldsymbol{\sigma}_{L}^{j}}{\partial X_{i}}=\frac{\partial \mathbf{C}_{L}^{j}}{\partial X_{i}} \cdot \mathbf{f}_{L}^{j}+\mathbf{C}_{L}^{j} \cdot \frac{\partial \mathbf{f}_{L}^{j}}{\partial X_{i}} .
$$

Using the well-known finite element theory, the internal forces vector $\mathbf{f}_{L}^{j}$ in the local coordinate system is given by

$$
\mathbf{f}_{L}^{j}=\mathbf{K}_{L}^{j} \cdot \mathbf{u}_{L}^{j}=\mathbf{K}_{L}^{j} \cdot \mathbf{T}_{L G}^{j} \cdot \mathbf{T}_{01}^{j} \cdot \mathbf{K}_{G}^{-1} \cdot \mathbf{f}_{G},
$$

where $\mathbf{K}_{G}$ is the global stiffness matrix (in the global coordinate system), $\mathbf{f}_{G}$ is the external nodal forces vector (in the global coordinate system), $\mathbf{T}_{L G}^{j}$ is a transformation matrix between the local and global coordinate systems, $\mathbf{T}_{01}^{j}$ is a Boolean matrix, i.e. the localization matrix determining the element position in the global stiffness matrix, it means

$$
\mathbf{u}_{G}^{j}=\mathbf{T}_{01}^{j} \cdot \mathbf{u}_{G} .
$$

Let's now realize the derivation of the internal nodal forces vector (7) with respect to $X_{i}$ :

$$
\begin{aligned}
& \frac{\partial \mathbf{f}_{L}^{j}}{\partial X_{i}}=\frac{\partial \mathbf{K}_{L}^{j}}{\partial X_{i}} \cdot \mathbf{u}_{L}^{j}+\mathbf{K}_{L}^{j} \cdot \frac{\partial \mathbf{u}_{L}^{j}}{\partial X_{i}}=\frac{\partial \mathbf{K}_{L}^{j}}{\partial X_{i}} \cdot \mathbf{T}_{L G}^{j} \cdot \mathbf{T}_{01}^{j} \cdot \mathbf{K}_{G}^{-1} . \\
& \mathbf{f}_{G}+\mathbf{K}_{L}^{j} \cdot \frac{\partial \mathbf{u}_{L}^{j}}{\partial X_{i}} .
\end{aligned}
$$

Applying the derivation on the well-known "FEA" equation $\mathbf{K}_{G} \cdot \mathbf{u}_{G}=\mathbf{f}_{G}$ we can write

$$
\frac{\partial \mathbf{K}_{G}}{\partial X_{i}} \cdot \mathbf{u}_{G}+\mathbf{K}_{G} \cdot \frac{\partial \mathbf{u}_{G}}{\partial X_{i}}=\frac{\partial \mathbf{f}_{G}}{\partial X_{i}} .
$$

where

$$
\frac{\partial \mathbf{K}_{G}}{\partial X_{i}}=\sum_{j=1}^{n i} \mathbf{T}_{01}^{j}{ }^{T} \cdot \mathbf{T}_{L G}^{j}{ }^{T} \cdot \frac{\partial \mathbf{K}_{L}^{j}}{\partial X_{i}} \cdot \mathbf{T}_{L G}^{j} \cdot \mathbf{T}_{01}^{j},
$$

and $n_{i}$ is a number of all the elements containing $X_{i}$. Further, the gradient of a global vector of the nodal displacements can be following

$$
\begin{aligned}
& \frac{\partial \mathbf{u}_{G}}{\partial X_{i}}=\mathbf{K}_{G}^{-1} \cdot\left(\frac{\partial \mathbf{f}_{G}}{\partial X_{i}}-\frac{\partial \mathbf{K}_{G}}{\partial X_{i}} \cdot \mathbf{u}_{G}\right)=\mathbf{K}_{G}^{-1} \cdot\left[\frac{\partial \mathbf{f}_{G}}{\partial X_{i}}-\right. \\
& \left.-\sum_{j=1}^{n i}\left(\mathbf{T}_{01}^{j T} \cdot \mathbf{T}_{L G}^{j}{ }^{T} \cdot \frac{\partial \mathbf{K}_{L}^{j}}{\partial X_{i}} \cdot \mathbf{T}_{L G}^{j} \cdot \mathbf{T}_{01}^{j}\right) \cdot \mathbf{u}_{G}\right]=\mathbf{K}_{G}^{-1} \cdot \\
& {\left[\frac{\partial \mathbf{f}_{G}}{\partial X_{i}}-\sum_{j=1}^{n i}\left(\mathbf{T}_{01}^{j T} \cdot \mathbf{T}_{L G}^{j}{ }^{T} \cdot \frac{\partial \mathbf{K}_{L}^{j}}{\partial X_{i}} \cdot \mathbf{T}_{L G}^{j} \cdot \mathbf{T}_{01}^{j}\right) \cdot \mathbf{K}_{G}^{-1} \cdot \mathbf{u}_{G}\right]}
\end{aligned}
$$

Relationship between $\mathbf{u}_{L}^{j}$ and $\mathbf{u}_{G}$ will be given by

$$
\begin{aligned}
& \frac{\partial \mathbf{u}_{L}^{j}}{\partial X_{i}}=\mathbf{T}_{L G}^{j} \cdot \frac{\partial \mathbf{u}_{G}^{j}}{\partial X_{i}}=\mathbf{T}_{L G}^{j} \cdot \mathbf{T}_{01}^{j} \cdot \frac{\partial \mathbf{u}_{G}}{\partial X_{i}}=\mathbf{T}_{L G}^{j} \cdot \mathbf{T}_{01}^{j} \cdot \mathbf{K}_{G}^{-1} \cdot \\
& \cdot\left[\frac{\partial \mathbf{f}_{G}}{\partial X_{i}}-\sum_{j=1}^{n i}\left(\mathbf{T}_{01}^{j T} \cdot \mathbf{T}_{L G}^{j}{ }^{T} \cdot \frac{\partial \mathbf{K}_{L}^{j}}{\partial X_{i}} \cdot \mathbf{T}_{L G}^{j} \cdot \mathbf{T}_{01}^{j}\right) \cdot \mathbf{K}_{G}^{-1} \cdot \mathbf{f}_{G}\right] .
\end{aligned}
$$

Substituting (13) into (9), we can obtain the derivation of the $\mathbf{f}_{L}^{j}$ with respect to $X_{i}$ as follows

$$
\begin{aligned}
& \frac{\partial \mathbf{f}_{L}^{j}}{\partial X_{i}}=\frac{\partial \mathbf{K}_{L}^{j}}{\partial X_{i}} \cdot \mathbf{T}_{L G}^{j} \cdot \mathbf{T}_{01}^{j} \cdot \mathbf{K}_{G}^{-1} \cdot \mathbf{f}_{G}+\mathbf{K}_{L}^{j} \cdot \mathbf{T}_{L G}^{j} \cdot \mathbf{T}_{01}^{j} \cdot \mathbf{K}_{G}^{-1} \cdot \\
& {\left[\frac{\partial \mathbf{f}_{G}}{\partial X_{i}}-\sum_{j=1}^{n i}\left(\mathbf{T}_{01}^{j T} \cdot \mathbf{T}_{L G}^{j}{ }^{T} \cdot \frac{\partial \mathbf{K}_{L}^{j}}{\partial X_{i}} \cdot \mathbf{T}_{L G}^{j} \cdot \mathbf{T}_{01}^{j}\right) \cdot \mathbf{K}_{G}^{-1} \cdot \mathbf{f}_{G}\right] .}
\end{aligned}
$$

Finally, after the substituting (14) into (6) we give the gradient of the $j$-th element stress vector

$$
\begin{aligned}
& \frac{\partial \boldsymbol{\sigma}_{L}^{j}}{\partial X_{i}}=\frac{\partial \mathbf{C}_{L}^{j}}{\partial X_{i}} \cdot \mathbf{K}_{L}^{j} \cdot \mathbf{T}_{L G}^{j} \cdot \mathbf{T}_{01}^{j} \cdot \mathbf{K}_{G}^{-1} \cdot \mathbf{f}_{G}+\mathbf{C}_{L}^{j} \cdot\left\{\frac{\partial \mathbf{K}_{L}^{j}}{\partial X_{i}} \cdot\right. \\
& \cdot \mathbf{T}_{L G}^{j} \cdot \mathbf{T}_{01}^{j} \cdot \mathbf{K}_{G}^{-1} \cdot \mathbf{f}_{G}+\mathbf{K}_{L}^{j} \cdot \mathbf{T}_{L G}^{j} \cdot \mathbf{T}_{01}^{j} \cdot \mathbf{K}_{G}^{-1} \cdot\left[\frac{\partial \mathbf{f}_{G}}{\partial X_{i}}-\right. \\
& \left.\left.-\sum_{j=1}^{n i}\left(\mathbf{T}_{01}^{j T} \cdot \mathbf{T}_{L G}^{j T} \cdot \frac{\partial \mathbf{K}_{L}^{j}}{\partial X_{i}} \cdot \mathbf{T}_{L G}^{j} \cdot \mathbf{T}_{01}^{j}\right) \cdot \mathbf{K}_{G}^{-1} \cdot \mathbf{f}_{G}\right]\right\} .
\end{aligned}
$$

It should be noted that the derivation of the matrix $C_{j}$ with respect to $X_{i}$, depends on the used cross-section. To realize the derivation $\frac{\partial \boldsymbol{\sigma}_{L}^{j}}{\partial X_{i}}$ means to find the following derivations $\frac{\partial \mathbf{K}_{L}^{j}}{\partial X_{i}}$, $\frac{\partial \mathbf{C}_{L}^{j}}{\partial X_{i}}$ and $\frac{\partial \mathbf{f}_{G}}{\partial X_{i}}$. The last derivation usually equals to zero or is not significant for the sensitivity analysis.

Let the cross-section area be variable $X_{i}$ then other cross-section characteristics will be expressed as follows

$$
J_{y}=a \cdot X_{i}^{p}, J_{z}=b \cdot X_{i}^{q}, J_{y}=a \cdot X_{i}^{r},
$$

where parameters $a, b, c$ and exponents $p, q, r$ will be obtained exactly (a simple cross-section) or numerically (a more complicated cross section), using the least squares method. The values of these parameters are presented in Tab. 1. 
Parameters $a, b, c$ and exponents $p, q, r$

Tab. 1 for chosen cross sections

\begin{tabular}{|c|c|c|c|}
\hline Cross-section & $J_{y}=a \cdot X_{i}^{p}$ & $J_{z}=b \cdot X_{i}^{q}$ & $J_{y}=a \cdot X_{i}^{r}$ \\
\hline \multirow{2}{*}{ Circle } & $a=0.0796$ & $b=0.0796$ & $c=0.1592$ \\
\hline & $p=2$ & $q=2$ & $r=2$ \\
\hline \multirow{2}{*}{ Square } & $a=0.0833$ & $b=0.0833$ & $c=0.14$ \\
\hline & $p=2$ & $q=2$ & $r=2$ \\
\hline \multirow{2}{*}{ I } & $a=1.4389$ & $b=0.7947$ & $c=0.0094$ \\
\hline & $p=2.0401$ & $q=1.7588$ & $r=2.0276$ \\
\hline \multirow{2}{*}{$=$} & $a=11.8364$ & $b=13.8364$ & $c=2.7273$ \\
\hline & $p=1.6047$ & $q=1.6217$ & $r=1.3592$ \\
\hline
\end{tabular}

The derivation $\frac{\partial \mathbf{K}_{L}^{j}}{\partial X_{i}}$ can be given by

$$
\begin{aligned}
& \frac{\partial \mathbf{K}_{L}^{j}}{\partial X_{i}}=\frac{\delta_{i j}}{X_{i}} \cdot\left[\mathbf{K}_{1}^{j}\left(X_{i}\right)+p \cdot \mathbf{K}_{2}^{j}\left(a \cdot X_{i}^{p}\right)+\right. \\
& \left.+q \cdot \mathbf{K}_{3}^{j}\left(b \cdot X_{i}^{q}\right)+r \cdot \mathbf{K}_{4}^{j}\left(c \cdot X_{i}^{r}\right)\right]
\end{aligned}
$$

where $\delta_{i j}$ is Kronecker delta and matrices $\mathbf{K}_{1,2,3,4}^{j}$ are sub-matrices of the stiffness matrix corresponding axial, bending (about axes $y$ and $\mathrm{z}$ ) and torsion stiffness of the used cross section (More information about stiffness parameters is in $[1,5,6])$.

Let's now focus on the derivation $\frac{\partial \mathbf{C}_{L}^{j}}{\partial X_{i}}$. The transformations matrix $\mathbf{C}_{L}^{j}$ depends on coordinates of the marginal points 1, 2, 3, 4, which have to be expressed by the variable $X_{i}$. Considering these conditions we can express matrix $\mathbf{C}_{L}^{j}$ for circular section as follows

$$
\mathbf{C}_{L}^{i}=\left\{\begin{array}{cccccccccccc}
0 & 0 & 0 & 0 & -\frac{4 \cdot \sqrt{\pi}}{\sqrt{X_{i}^{3}}} & 0 & \frac{1}{X_{i}} & 0 & 0 & 0 & 0 & 0 \\
0 & 0 & 0 & 0 & 0 & \frac{4 \cdot \sqrt{\pi}}{\sqrt{X_{i}^{3}}} & \frac{1}{X_{i}} & 0 & 0 & 0 & 0 & 0 \\
0 & 0 & 0 & 0 & \frac{4 \cdot \sqrt{\pi}}{\sqrt{X_{i}^{3}}} & 0 & \frac{1}{X_{i}} & 0 & 0 & 0 & 0 & 0 \\
0 & 0 & 0 & 0 & 0 & -\frac{4 \cdot \sqrt{\pi}}{\sqrt{X_{i}^{3}}} & \frac{1}{X_{i}} & 0 & 0 & 0 & 0 & 0 \\
0 & 0 & 0 & 0 & 0 & 0 & \frac{1}{X_{i}} & 0 & 0 & 0 & -\frac{4 \cdot \sqrt{\pi}}{\sqrt{X_{i}^{3}}} & 0 \\
0 & 0 & 0 & 0 & 0 & 0 & \frac{1}{X_{i}} & 0 & 0 & 0 & 0 & \frac{4 \cdot \sqrt{\pi}}{\sqrt{X_{i}^{3}}} \\
0 & 0 & 0 & 0 & 0 & 0 & \frac{1}{X_{i}} & 0 & 0 & 0 & \frac{4 \cdot \sqrt{\pi}}{\sqrt{X_{i}^{3}}} & 0 \\
0 & 0 & 0 & 0 & 0 & 0 & \frac{1}{X_{i}} & 0 & 0 & 0 & 0 & -\frac{4 \cdot \sqrt{\pi}}{\sqrt{X_{i}^{3}}}
\end{array}\right\}
$$

and the analyzed derivation of this matrix gets the following form

$$
\frac{\partial \mathbf{C}_{L}^{i}}{\partial X_{i}}=\left\{\begin{array}{cccccccccccc}
0 & 0 & 0 & 0 & \frac{6 \cdot \sqrt{\pi}}{\sqrt{X_{i}^{5}}} & 0 & -\frac{1}{X_{i}^{2}} & 0 & 0 & 0 & 0 & 0 \\
0 & 0 & 0 & 0 & 0 & -\frac{6 \cdot \sqrt{\pi}}{\sqrt{X_{i}^{5}}} & -\frac{1}{X_{i}^{2}} & 0 & 0 & 0 & 0 & 0 \\
0 & 0 & 0 & 0 & -\frac{6 \cdot \sqrt{\pi}}{\sqrt{X_{i}^{5}}} & 0 & -\frac{1}{X_{i}^{2}} & 0 & 0 & 0 & 0 & 0 \\
0 & 0 & 0 & 0 & 0 & \frac{6 \cdot \sqrt{\pi}}{\sqrt{X_{i}^{5}}} & -\frac{1}{X_{i}^{2}} & 0 & 0 & 0 & 0 & 0 \\
0 & 0 & 0 & 0 & 0 & 0 & -\frac{1}{X_{i}^{2}} & 0 & 0 & 0 & \frac{6 \cdot \sqrt{\pi}}{\sqrt{X_{i}^{5}}} & 0 \\
0 & 0 & 0 & 0 & 0 & 0 & -\frac{1}{X_{i}^{2}} & 0 & 0 & 0 & 0 & 0 \\
0 & 0 & 0 & 0 & 0 & 0 & -\frac{1}{X_{i}^{2}} & 0 & 0 & 0 & -\frac{6 \cdot \sqrt{\pi}}{\sqrt{X_{i}^{5}}} & 0 \\
0 & 0 & 0 & 0 & 0 & 0 & -\frac{1}{X_{i}^{2}} & 0 & 0 & 0 & 0 & \frac{6 \cdot \sqrt{\pi}}{\sqrt{X_{i}^{5}}}
\end{array}\right\}
$$

It is possible to get exactly the previous matrices but, for example, $\mathbf{C}_{L}^{j}$ of the I-section has to be analyzed numerically [6].

\section{Example 1}

Let us consider the structural sensitivity analysis of normal stresses of the beam element from Fig. 2. Given: $E=2.1 \mathrm{e} 5 \mathrm{MPa}$ $F_{1}=800 \mathrm{~N}, F_{2}=6000 \mathrm{~N}, A_{1}=200 \mathrm{~mm}^{2}, A_{2}=35 \mathrm{~mm}^{2}$, $L_{1}=1 \mathrm{~m}, L_{2}=0.5 \mathrm{~m}$.

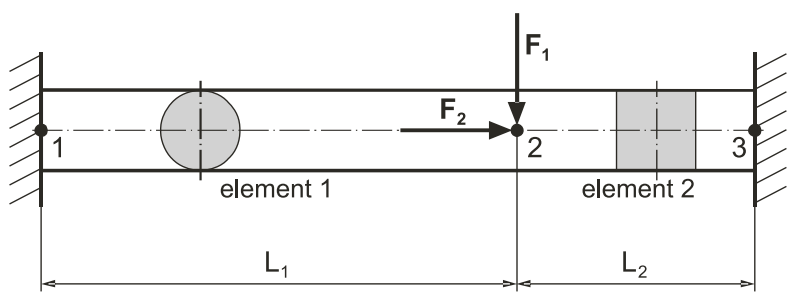

Fig. 2 Simple beam structure with 2 cross section 
Applying previous relationships we can obtain the following derivations of matrices $\mathbf{C}_{L}^{1}$ and $\mathbf{C}_{L}^{2}$

$$
\frac{\partial \mathbf{C}_{L}^{1}}{\partial X_{1}}=\left\{\begin{array}{cccrcc}
0 & 0 & 0 & -2.5 e-5 & 0 & 0 \\
0 & 0 & -1.8 e-5 & -2.5 e-5 & 0 & 0 \\
0 & 0 & 0 & -2.5 e-5 & 0 & 0 \\
0 & 0 & -1.8 e-5 & -2.5 e-5 & 0 & 0 \\
0 & 0 & 0 & -2.5 e-5 & 0 & 0 \\
0 & 0 & 0 & -2.5 e-5 & 0 & -1.8 e-5 \\
0 & 0 & 0 & -2.5 e-5 & 0 & 0 \\
0 & 0 & 0 & -2.5 e-5 & 0-1.8 e-5
\end{array}\right\},
$$

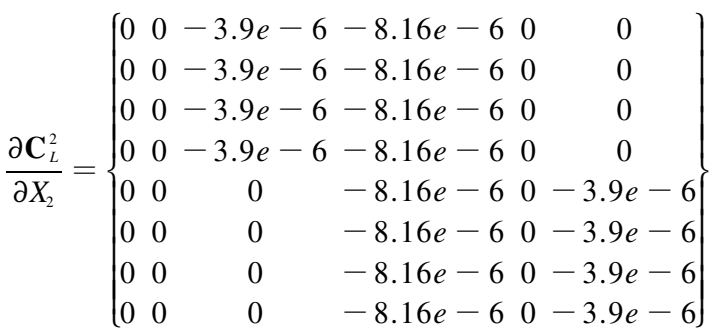

and finally stress gradients in marginal points of the used crosssections are the following

$$
\frac{\partial \sigma_{L}^{(1)}}{\partial A_{1}}=\left\{\begin{array}{c}
-0,0222 \\
-0,8077 \\
-0,0222 \\
0,7632 \\
-0,0222 \\
-1,1897 \\
-0,0222 \\
1,1452
\end{array}\right\} \text { and } \frac{\partial \sigma_{L}^{(1)}}{\partial A_{1}}=\left\{\begin{array}{c}
-0,0222 \\
-0,8077 \\
-0,0222 \\
0,7632 \\
-0,0222 \\
-1,1897 \\
-0,0222 \\
1,1452
\end{array}\right\}\left[\mathrm{MPa} / \mathrm{mm}^{2}\right]
$$

The graphic presentation of the stress gradients is in Fig. 3. The presented stress gradient analysis was confronted with a "classical" numerical computational approach $\left(\Delta \sigma j / \Delta X_{i}\right)$ and it's possible to observe the absolute consensus.

\section{Stress sensitivity analysis for a thin shell finite element}

The finite element modeling of box, shell or thin-walled structures are usually realized using thin shell finite elements (Kirchhoff's or Mindlin's formulation) [1, 4, 9]. The stiffness parameters
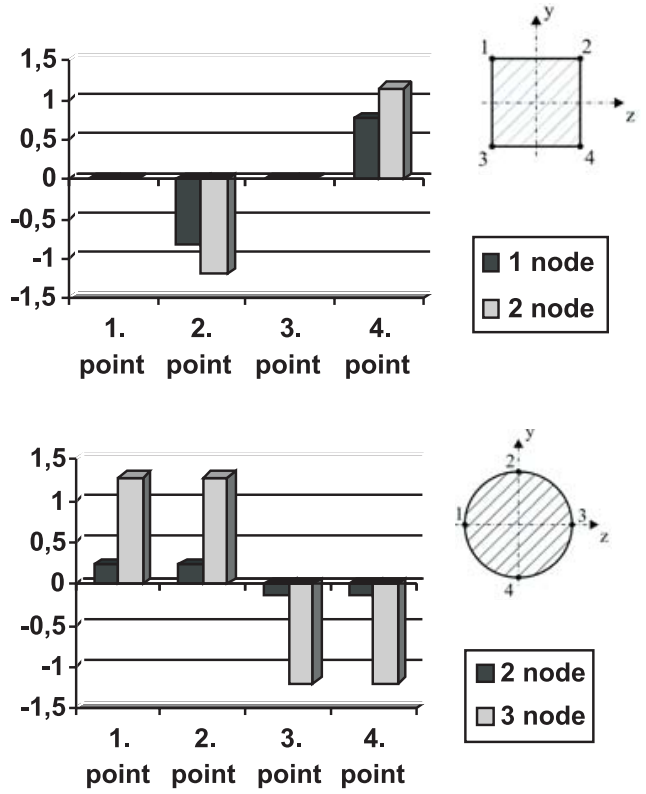

Fig. 3 Values of the stress gradient in the first and second element

depend on material constants and element geometry, mainly on its thickness. Therefore, the thickness $t_{i}$ will be the variable in the following theoretical and numerical stress sensitivity analysis of the shell finite element; the fundamental information about this analysis can be found in $[1,4,9]$.

At first we have to prepare the stress calculation process. This process is based on the expression of the $j$-th element membrane forces and bending moments (without shear forces) [4, 6], i.e.

$$
\begin{aligned}
& {\left[\begin{array}{lll}
F_{x x} & F_{y y} & F_{x y}
\end{array}\right]_{j}^{T}=\mathbf{F}_{m}^{j}=\int_{S} \mathbf{E}_{m}^{j} \cdot \varepsilon_{m}^{j} d S_{j}=} \\
& =\mathbf{E}_{m}^{j} \cdot \int_{S} \mathbf{B}_{m}^{j} d S_{j} \cdot \mathbf{u}_{L}^{j}=t_{j} \cdot \mathbf{D}_{j} \cdot \mathbf{I}_{m}^{j} \cdot \mathbf{u}_{L}^{j}
\end{aligned}
$$

and

$$
\begin{aligned}
& {\left[M_{x x} M_{y y} M_{x y}\right]_{j}^{T}=\mathbf{M}_{b}^{j}=\int_{S} \mathbf{E}_{b}^{j} \cdot \varepsilon_{b}^{j} d S_{j}=} \\
& =\mathbf{E}_{b}^{j} \cdot \int_{S} \mathbf{B}_{b}^{j} d S_{j} \cdot \mathbf{u}_{L}^{j}=\frac{t_{j}^{3}}{12} \cdot \mathbf{D}_{j} \cdot \mathbf{I}_{b}^{j} \cdot \mathbf{u}_{L}^{j} .
\end{aligned}
$$

The auxiliary matrices $\mathbf{I}_{m}$ and $\mathbf{I}_{b}$ can be calculated only using the numerical approach. Further details about $\mathbf{E}_{m}, \mathbf{E}_{b}, \mathbf{D}, \mathbf{B}_{m}, \mathbf{B}_{b}$, $\mathbf{u}_{e l}$ and $t$ are presented in [6]. The extreme stress values can be expected at the top or at the bottom surface. Generally, it means or in compliance with the previous beam element (eq. 5)

$$
\left[\begin{array}{ll}
\sigma_{m b} \mid{ }_{t o p} \\
\left.\sigma_{m b}\right|_{b o t} ^{j}
\end{array}\right]^{j}=\left\{\begin{array}{l}
\sigma_{x x, t o p} \\
\sigma_{y y, t o p} \\
\sigma_{x y, t o p} \\
\sigma_{x x, b o t} \\
\sigma_{y y, b o t} \\
\sigma_{x y, b o t}
\end{array}\right\}=\left\{\begin{array}{cccccc}
1 / t_{j} & 0 & 0 & 6 / t_{j}^{2} & 0 & 0 \\
0 & 1 / t_{j} & 0 & 0 & 6 / t_{j}^{2} & 0 \\
0 & 0 & 1 / t_{j} & 0 & 0 & 6 / t_{j}^{2} \\
1 / t_{j} & 0 & 0 & 6 / t_{j}^{2} & 0 & 0 \\
0 & 1 / t_{j} & 0 & 0 & 6 / t_{j}^{2} & 0 \\
0 & 0 & 1 / t_{j} & 0 & 0 & 6 / t_{j}^{2}
\end{array}\right\} \cdot\left\{\begin{array}{l}
F_{x x} \\
F_{y y} \\
F_{x y} \\
M_{x x} \\
M_{y y} \\
M_{x y}
\end{array}\right\}=\left[\begin{array}{l}
\mathbf{A}_{t, t o p} \\
\mathbf{A}_{t, b o t}
\end{array}\right]^{j} \cdot\left\{\begin{array}{l}
\mathbf{F}_{m} \\
\mathbf{M}_{b}
\end{array}\right\}^{j},
$$


$\boldsymbol{\sigma}_{m b_{-} L}^{j}=\mathbf{C}_{L}^{j} \cdot \mathbf{f}_{L}^{j}$.

Let's build new material and auxiliary matrices

$$
\mathbf{E}_{m b}=\left[\begin{array}{cc}
t_{j} \cdot \mathbf{I}_{3} & 0_{3} \\
0_{3} & \frac{t_{j}^{3}}{12} \cdot \mathbf{I}_{3}
\end{array}\right] \cdot\left\{\begin{array}{l}
\mathbf{D} \\
\mathbf{D}
\end{array}\right\}_{j}=\mathbf{D}_{t} \cdot \mathbf{D}_{m b}, \mathbf{I}_{m b}=\left\{\begin{array}{c}
\mathbf{I}_{m}^{j} \\
\mathbf{I}_{b}^{j}
\end{array}\right\},
$$

where the matrix $\mathbf{I}_{3}$ is the classical unit matrix. Then (22) can be written as follows

$$
\begin{aligned}
& \left.\boldsymbol{\sigma}_{j \_m b}\right|_{t o p}=\mathbf{A}_{t, t o p} \cdot \mathbf{E}_{m b} \cdot \mathbf{I}_{m b} \cdot \mathbf{u}_{L}^{j}=\mathbf{A}_{t, t o p} \cdot \mathbf{D}_{t} \cdot \mathbf{D}_{m b} \cdot \mathbf{I}_{m b} \cdot \mathbf{u}_{L}^{j},(25 \mathrm{a}) \\
& \left.\boldsymbol{\sigma}_{j, m b}\right|_{b o t}=\mathbf{A}_{t, b o t} \cdot \mathbf{E}_{m b} \cdot \mathbf{I}_{m b} \cdot \mathbf{u}_{L}^{j}=\mathbf{A}_{t, b o t} \cdot \mathbf{D}_{t} \cdot \mathbf{D}_{m b} \cdot \mathbf{I}_{m b} \cdot \mathbf{u}_{L}^{j} \cdot(25 \mathrm{~b})
\end{aligned}
$$

Generally, the top or bottom von Mises stresses may be calculated from relations

or

$$
\left.\sigma_{j_{e} e k v}^{2}\right|_{t o p}=\left.\left.\boldsymbol{\sigma}_{j_{\_} m b}^{T}\right|_{t o p} \cdot \mathbf{T}_{m b} \cdot \boldsymbol{\sigma}_{j_{\perp} m b}\right|_{t o p}
$$$$
\left.\sigma_{j \_e k v}^{2}\right|_{b o t}=\left.\left.\boldsymbol{\sigma}_{j \_m b}^{T}\right|_{b o t} \cdot \mathbf{T}_{m b} \cdot \boldsymbol{\sigma}_{j \_m b}\right|_{b o t}
$$

where

$$
T_{m b}=\left[\begin{array}{ccc}
1 & -0.5 & 0 \\
-0.5 & 1 & 0 \\
0 & 0 & 3
\end{array}\right] .
$$

Using (25) and (27) in (26) we obtain

$$
\left.\boldsymbol{\sigma}_{j_{-} e k v}^{2}\right|_{t o p}=\left.\left.\boldsymbol{\sigma}_{j_{\_} m b}^{T}\right|_{t o p} \cdot \mathbf{T}_{m b} \cdot \boldsymbol{\sigma}_{j_{-} m b}\right|_{\text {top }}=
$$$$
=\mathbf{u}_{L}^{j T} \cdot \mathbf{I}_{m b}^{T} \cdot \mathbf{D}_{m b}^{T} \cdot \mathbf{D}_{t}^{T} \cdot \mathbf{A}_{t, t o p}^{T} \cdot \mathbf{T}_{m b} \cdot \mathbf{A}_{t, t o p} \cdot \mathbf{D}_{t} \cdot \mathbf{D}_{m b} \cdot \mathbf{I}_{m b} \cdot \mathbf{u}_{L}^{j}=(28 \mathrm{a})
$$$$
=\mathbf{u}_{L}^{j T} \cdot \mathbf{I}_{m b}^{T} \cdot \mathbf{D}_{m b}^{T} \cdot \mathbf{D}_{m b} \cdot \mathbf{I}_{m b} \cdot \mathbf{u}_{L}^{j}
$$

and

$$
\begin{aligned}
& \left.\sigma_{j_{e} e k v}^{2}\right|_{b o t}=\boldsymbol{\sigma}_{j_{j} m b}^{T}\left|{ }_{b o t} \cdot \mathbf{T}_{m b} \cdot \boldsymbol{\sigma}_{j \_m b}\right|_{b o t}= \\
& =\mathbf{u}_{L}^{j T} \cdot \mathbf{I}_{m b}^{T} \cdot \mathbf{D}_{m b}^{T} \cdot \mathbf{D}_{t}^{T} \cdot \mathbf{A}_{t, b o t}^{T} \cdot \mathbf{T}_{m b} \cdot \mathbf{A}_{t, b o t} \cdot \mathbf{D}_{t} \cdot \mathbf{D}_{m b} \cdot \mathbf{I}_{m b} \cdot \mathbf{u}_{L}^{j}=(28 \mathrm{~b}) \\
& =\mathbf{u}_{L}^{j T} \cdot \mathbf{I}_{m b}^{T} \cdot \mathbf{D}_{m b}^{T} \cdot \mathbf{D}_{m b} \cdot \mathbf{I}_{m b} \cdot \mathbf{u}_{L}^{j}
\end{aligned}
$$

where

and

$$
T_{t, \text { top }}=\left[\begin{array}{cccccc}
1 & -0.5 & 0 & 0.5 \cdot t_{j} & -0.25 \cdot t_{j} & 0 \\
-0.5 & 1 & 0 & -0.25 \cdot t_{j} & 0.5 \cdot t_{j} & 0 \\
0 & 0 & 3 & 0 & 0 & 1.5 \cdot t_{j} \\
0.5 \cdot t_{j} & -0.25 \cdot t_{j} & 0 & 0.25 \cdot t_{j}^{2} & -0.125 \cdot t_{j}^{2} & 0 \\
-0.25 \cdot t_{j} & 0.5 \cdot t_{j} & 0 & -0.125 \cdot t_{j}^{2} & 0.25 \cdot t^{2} & 0 \\
0 & 0 & 1.5 \cdot t_{j} & 0 & 0 & 0.75 \cdot t_{j}^{2}
\end{array}\right]
$$

$$
T_{t, b o t}=\left[\begin{array}{cccccc}
1 & -0.5 & 0 & -0.5 \cdot t_{j} & 0.25 \cdot t_{j} & 0 \\
-0.5 & 1 & 0 & 0.25 \cdot t_{j} & -0.5 \cdot t_{j} & 0 \\
0 & 0 & 3 & 0 & 0 & -1.5 \cdot t_{j} \\
-0.5 \cdot t_{j} & 0.25 \cdot t_{j} & 0 & 0.25 \cdot t_{j}^{2} & -0.125 \cdot t_{j}^{2} & 0 \\
0.25 \cdot t_{j} & -0.5 \cdot t_{j} & 0 & -0.125 \cdot t_{j}^{2} & 0.25 \cdot t_{j}^{2} & 0 \\
0 & 0 & -1.5 \cdot t_{j} & 0 & 0 & 0.75 \cdot t_{j}^{2}
\end{array}\right]
$$




$$
\begin{aligned}
& \frac{\left.\partial \sigma_{j \_e k v}^{2}\right|_{b o t}}{\partial t_{i}}=\frac{\partial \mathbf{u}^{T}}{\partial t_{i}} \cdot \mathbf{T}_{j_{-} 01}^{T} \cdot \mathbf{T}_{j \_L G}^{T} \cdot \mathbf{I}_{j_{-} m b}^{T} \cdot \mathbf{D}_{j_{-} m b}^{T} \cdot \mathbf{T}_{j_{-}, b o t} \cdot \\
& \cdot \mathbf{D}_{j_{\_} m b} \cdot \mathbf{I}_{j_{j} m b} \cdot \mathbf{T}_{v L G} \cdot \mathbf{T}_{j_{-} 01} \cdot \mathbf{u}+\mathbf{u}^{T} \cdot \mathbf{T}_{j_{-} 01}^{T} \cdot \mathbf{T}_{j_{\perp} L G}^{T} \cdot \mathbf{I}_{j_{-} m b}^{T} \cdot \\
& \cdot \mathbf{D}_{j_{-} m b}^{T} \cdot \delta_{i j} \cdot \frac{\partial \mathbf{T}_{j_{-} t, b o t}}{\partial t_{i}} \cdot D_{j_{-} m b} \cdot \mathbf{I}_{j_{-} m b} \cdot \mathbf{T}_{j_{L} L G} \cdot \mathbf{T}_{j_{-} 01} \cdot \mathbf{u}+ \\
& +\mathbf{u}^{T} \cdot \mathbf{T}_{j_{-} 01}^{T} \cdot \mathbf{T}_{j_{-} L G}^{T} \cdot \mathbf{I}_{j_{-} m b}^{T} \cdot \mathbf{D}_{j_{-} m b}^{T} \cdot \mathbf{T}_{j_{-}, t b o t} \cdot \mathbf{D}_{j_{-} m b} \cdot \mathbf{I}_{j_{-} m b} \cdot \\
& \cdot \mathbf{T}_{j L L G} \cdot \mathbf{T}_{j \_01} \cdot \frac{\partial \mathbf{u}}{\partial t_{i}},
\end{aligned}
$$

where

$$
\begin{aligned}
& \frac{\left.\partial \sigma_{j_{e} e k v}^{2}\right|_{t o p}}{\partial t_{i}}=\frac{1}{\left.2 \sigma_{j_{-} e k v}\right|_{t o p}} \cdot \frac{\left.\partial \sigma_{j_{e} e k v}^{2}\right|_{t o p}}{\partial t_{i}} \text { and } \\
& \frac{\left.\partial \sigma_{j_{e} e k v}\right|_{b o t}}{\partial t_{i}}=\frac{1}{\left.2 \sigma_{j_{-} e k v}\right|_{b o t}} \cdot \frac{\left.\partial \sigma_{j_{e} e k v}^{2}\right|_{b o t}}{\partial t_{i}} .
\end{aligned}
$$

All the presented approaches have been implemented into Matlab's FE software MATFEM developed by the authors.

\section{Example 2}

Determine the element stress derivative (eqs. 32a, 32b) with respect to the thickness $t_{1}$ and $t_{2}$ of the shell structure in Fig. 4 .

and

$$
\frac{\partial \mathbf{T}_{j_{-}, t, o p}}{\partial t_{i}}=\delta_{i j} \cdot\left[\begin{array}{cccccc}
0 & 0 & 0 & 0.5 & -0.25 & 0 \\
0 & 0 & 0 & -0.25 & 0.5 & 0 \\
0 & 0 & 0 & 0 & 0 & 1.5 \\
0.5 & -0.25 & 0 & 0.5 \cdot t_{j} & -0.25 \cdot t_{j} & 0 \\
-0.25 & 0.5 & 0 & -0.25 \cdot t_{j} & 0.5 \cdot t_{j} & 0 \\
0 & 0 & 1,5 & 0 & 0 & 1.5 \cdot t_{j}
\end{array}\right]
$$

$$
\frac{\partial \mathbf{T}_{j_{-}, b o t}}{\partial t_{i}}=\delta_{i j} \cdot\left[\begin{array}{cccccc}
0 & 0 & 0 & -0,5 & 0,25 & 0 \\
0 & 0 & 0 & 0,25 & -0,5 & 0 \\
0 & 0 & 0 & 0 & 0 & -1,5 \\
-0,5 & 0,25 & 0 & 0,5 \cdot t_{j} & -0,25 \cdot t_{j} & 0 \\
0,25 & -0,5 & 0 & -0,25 \cdot t_{j} & 0,5 \cdot t_{j} & 0 \\
0 & 0 & -1,5 & 0 & 0 & 1,5 \cdot t_{j}
\end{array}\right]
$$

The derivative $u$ with respect to ti may be expressed as

$$
\frac{\partial \mathbf{u}_{G}}{\partial t_{i}}=\mathbf{K}_{G}^{-1} \cdot\left(\frac{\partial \mathbf{f}}{\partial t_{i}}-\frac{\partial \mathbf{K}_{G}}{\partial t_{i}} \cdot \mathbf{u}_{G}\right)
$$

or, in more detail,

$$
\begin{aligned}
& \frac{\partial \mathbf{u}_{G}}{\partial t_{i}}=\mathbf{K}_{G}^{-1} \cdot\left[\frac{\partial \mathbf{f}}{\partial t_{i}}-\left(\sum_{j=1}^{n} \mathbf{T}_{j_{-} 01}^{T} \cdot \mathbf{T}_{j \_L G}^{T} \cdot\right.\right. \\
& \left.\left.\frac{\partial\left(\mathbf{K}_{j \_m}+\mathbf{K}_{j \_b}+\mathbf{K}_{j \_s}\right)}{\partial t_{i}} \cdot \mathbf{T}_{j \_L G} \cdot \mathbf{T}_{j \_01}\right) \cdot \mathbf{u}_{G}\right]
\end{aligned}
$$

The relation $\frac{\partial \mathbf{f}}{\partial t_{i}}$ is often zero and the derivative of the all the element components of the stiffness matrix can be realized as follows [6]

$$
\frac{\partial\left(\mathbf{K}_{j_{-} m}+\mathbf{K}_{j_{-} b}+\mathbf{K}_{j \_s}\right)}{\partial t_{i}}=\frac{\delta_{i j}}{t_{i}} \cdot\left(\mathbf{K}_{j \_m}+3 \cdot \mathbf{K}_{j \_b}+\mathbf{K}_{j_{-} s}\right)
$$

The particular membrane, bending and shear matrices are presented in $[1,7]$.

Finally, the derivative of the von Mises stress (at the top and at the bottom surfaces) with respect to the element thickness $t_{i}$ is the following
Let's consider the following input parameters: elasticity modulus $E=3.106 \mathrm{MPa}$, Poisson's ratio $\mu=0.3$, thicknesses $t_{1}=3 \mathrm{~mm}$ and $t_{2}=2 \mathrm{~mm}$ and force $F_{Z}=2500 \mathrm{~N}$ concentrated into each node of the top curved surface.

Stress gradient values for the chosen elements - analytical vs. numerical calculation

\begin{tabular}{|c|c|c|c|c|c|}
\hline \multirow{2}{*}{$\begin{array}{c}\text { Nr. of } \\
\text { element }\end{array}$} & \multicolumn{2}{|c|}{$\begin{array}{c}\text { Stress gradient with } \\
\text { respect } t_{1}\end{array}$} & \multirow{2}{*}{$\begin{array}{c}\text { Nr. of } \\
\text { element }\end{array}$} & \multicolumn{2}{|c|}{$\begin{array}{c}\text { Stress gradient with } \\
\text { respect } t_{2}\end{array}$} \\
\cline { 6 - 7 } & Analytically & Numerically & & Analytically & Numerically \\
\hline 4 & 180.6925 & 180.8217 & 81 & 72.1432 & 72.1356 \\
\hline 15 & 178.1929 & 178.3464 & 66 & 56.8617 & 56.8841 \\
\hline 12 & 172.7673 & 172.9401 & 65 & 56.5136 & 56.5514 \\
\hline 7 & 172.2105 & 172.3427 & 92 & 54.8065 & 54.8449 \\
\hline 53 & 170.2041 & 170.4455 & 80 & 52.5394 & 52.5649 \\
\hline
\end{tabular}

The chosen calculated values of the stress gradients are written in Table 2. The presented analytic stress gradient calculation was confronted with the classical numerical computational approach $\left(\Delta \sigma_{j} / \Delta t_{i}\right)$. A graphic presentation of the stress gradients distribution in each of the elements is in Figs. 5 and 6. 
The results document the influence of both parameters on the stresses and the major signification of thickness $t_{1}$. This information may be used for the next optimizing process.

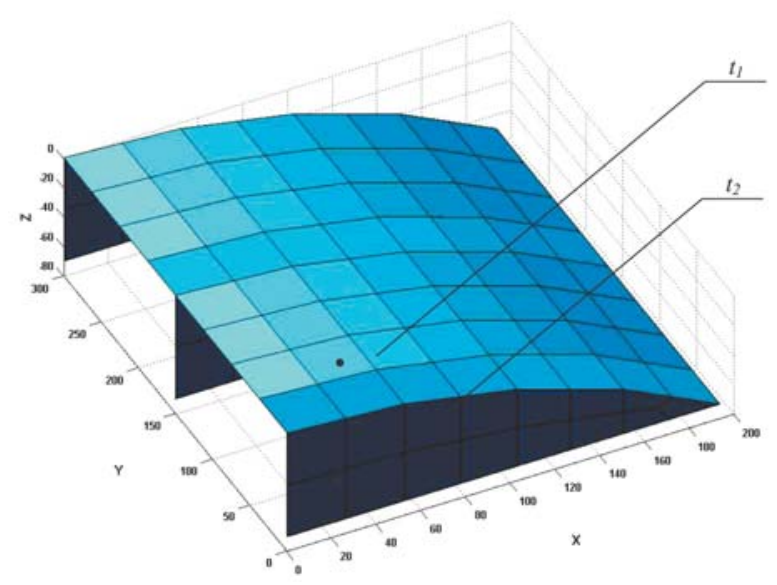

Fig. 4 Half model of the analysed shell structure in MATFEM

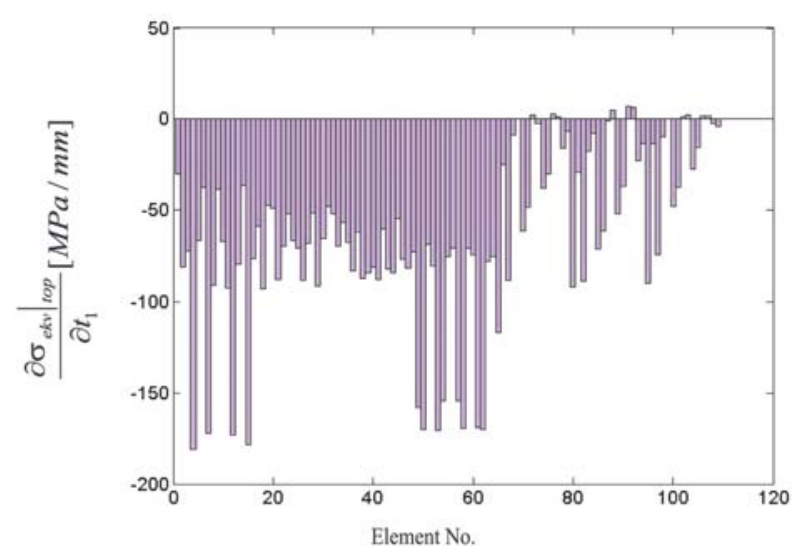

Fig. 5 Stress sensitivity with respect to $t_{1}$

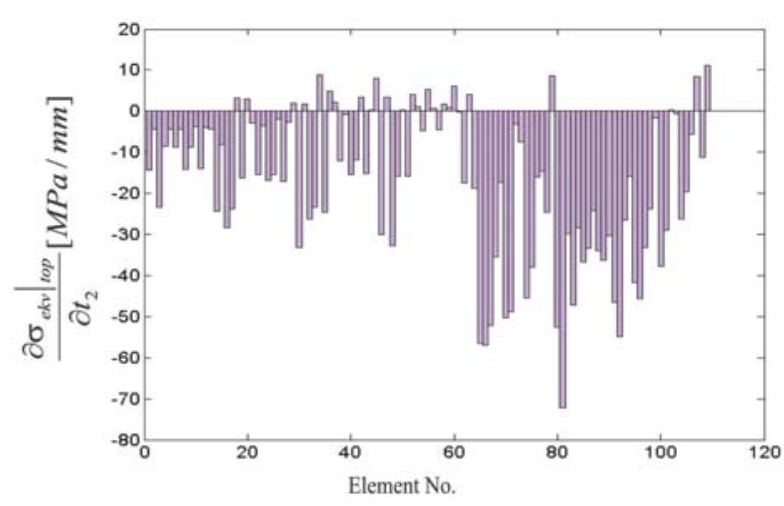

Fig. 6 Stress sensitivity with respect to $t_{2}$

\section{Conclusion}

The presented work deals with the theoretical aspects and numerical realization of the stress sensitivity analysis of the beam and shell finite elements focused on its cross section parameters (the area in the case of the beam element and the thickness in the case of the thin shell element). The whole computational procedure was inbuilt into Matlab's software module MATFEM. Testing examples support the authors' considerations about effectiveness of the proposed method.

\section{Acknowledgements}

This work has been supported by VEGA grant No. 1/4099/07 and by the research project AV 4/2044/08.

\section{References}

[1] BATHE, K. J.: Finite Element Procedures. New Persey, Prentice Hall, 1996.

[2] DEKÝŠ, V., SAPIETOVÁ, A., KOCÚR, R.: On the reliability estimation of the conveyer mechanism using the Monte Carlo method, Proc. of COSIM2006, Krynica-Zdroj, 2006, pp. 67-74.

[3] HAFTKA, R. T., GURDAL, Z.: Elements of Structural Optimization, Kluwer Academic Publisher, 1992.

[4] KWON, Y. W., BANG, H.: The Finite Element Method using MATLAB, CRC Press University of Minnesota, 1996.

[5] MURIN, J.: Finite Element Method for Bar Constructions and Framed Structures (in Slovak), STU, Bratislava 1999.

[6] SAGA, M., VASKO, M., KOCUR, R., TOTH, L., KOHAR, R.: Application of optimizing algorithms in solid mechanics. In Slovak, VTS, University of Zilina, 2006.

[7] M. SAGA A, M. VAAKO A, J. JANDACKA, Z. HOLKOVA: Contribution to stress sensitivity analysis, of the shell finite elements, Applied and Computational Mechanics, Vol. II, No.1, 2008, pp. 113-122 


\section{COMMNICOIIONS}

[8] SEGLA, S., CIUPITU, L., REICH, S.: Optimization of a spring balancing mechanism for parallelogram robot mechanisms, 1st International Conference "Optimization of robots and Manipulators OPTIROB 2006”, Predeal, Romania, 2006, pp. 69-74.

[9] VAVRO, J.: Optimization of the design of cross-sectional quantities in transport machines and equipment, Studia i materially, Zelena Hora, Poland, 1998, pp. 186-194.

[10] ZIENKIEWICZ, O. C.: The Finite Element Method in Engineering Science, McGraw, Hill, New York, 1971. 